\title{
Whole-Exome Sequencing
}

National Cancer Institute ( $\mathrm{NCl})$

\section{Source}

National Cancer Institute (NCI). Whole-Exome Sequencing.

A laboratory process that is used to determine the nucleotide sequence primarily of the exonic (or protein-coding) regions of an individual's genome and related sequences, representing approximately $1 \%$ of the complete DNA sequence. 